\title{
AUDIT, AUDIT-C Y AR2I PARA EVALUAR EL BINGE DRINKING EN UNIVERSITARIOS ESPAÑOLES
}

\section{AUDIT, AUDIT-C AND AR2I TO EVALUATE BINGE DRINKING IN SPANISH COLLEGE STUDENTS}

Maria Botella López, José Antonio Giménez Costa y María Teresa Cortés Tomás Universidad de Valencia. España

\section{Abstract}

The identification of young binge drinkers (BD) is a priority in the area of prevention and intervention, given the biopsycho-social consequences this phenomenon generates. Objective. Aim of the study is to verify which version of the AUDIT is best suited as a screening instrument for this population, based on an accurate operationalization of the BD. Method. A convenience sampling was carried out with 190 young alcohol consumers (73.7\% women), between 18 and 22 years old, who completed a self-report of alcohol consumption, the AUDIT and AR2I. Results. The cluster shows 5 groups of young BD, depending on the age of onset in consumption and the grams of alcohol ingested in a BD episode. After comparing the effectiveness of the different versions of AUDIT, the combination of items 2 and 3 reviewed (AR2I) is distinguished as the best option for the identification of this population. Conclusions. AR2I is a useful, fast and simple option to identify young BD, allowing its application in different contexts, such as Primary Care, Emergency Services and specialized services in drug prevention.

Keywords: AUDIT, binge drinking, screening, young people.

\section{Resumen}

La identificación de los jóvenes que realizan un consumo intensivo de alcohol ( $\mathrm{CIA}$ ) es una prioridad en el ámbito de la prevención e intervención, dadas las consecuencias bio-psico-sociales que genera. Objetivo. Verificar qué versión del AUDIT constituye el instrumento de screening más adecuado en esta población, partiendo de una operacionalización más precisa del CIA. Método. Se ha realizado un muestreo de conveniencia en el que han participado 190 jóvenes consumidores de alcohol entre 18 y 22 años (73.7\% mujeres), que cumplimentaron un autoregistro de consumo de alcohol, el AUDIT y AR2I. Resultados. El análisis de conglomerados por sexos muestra 5 grupos de jóvenes CIA, en función de la edad de inicio en el consumo de alcohol y los gramos ingeridos de esta sustancia durante un episodio CIA. De entre las versiones del AUDIT analizadas, la combinación de los ítems 2 y 3 revisados (AR2I) se desmarca como la mejor opción para identificar a este colectivo. Conclusiones. El AR2I resulta la opción más útil, rápida y sencilla de identificación de jóvenes CIA, permitiendo su implementación en contextos como Atención Primaria, Servicios de Urgencias y recursos especializados en prevención de drogas.

Palabras clave: AUDIT, consumo intensivo de alcohol, cribado, jóvenes. 
El Binge Drinking (BD) o Consumo Intensivo de Alcohol (CIA) es una práctica frecuente entre los jóvenes europeos (EDADES-Encuesta sobre Alcohol y Drogas en España-PNSD, 2019; ESPAD- European School Survey Project on Alcohol and other Drugs-, 2016; ESTUDESEncuesta Sobre Uso de Drogas en Enseñanzas Secundarias en España-PNSD, 2018; Eurobarómetro, 2010) que se asocia con importantes consecuencias biopsicosociales como bajo rendimiento académico o profesional, lagunas de memoria, resaca y dolor de cabeza, problemas interpersonales, conductas sexuales de riesgo o no planificadas, conducción bajo los efectos del alcohol, peleas, lesiones, tener problemas con la justicia o causar daños a terceros (Cortés, 2010; López et al., 2014; Mallett et al., 2011; Motos, 2013; Shield, Gmel, Patra, \& Rehm, 2012; Wechsler \& Nelson, 2001).

La variedad y la importancia de algunas de estas consecuencias, tanto a corto como a largo plazo (Cortés, Motos \& Giménez, 2015; López et al., 2014; Martínez, Sher, \& Wood, 2014; Shield et al., 2012) junto con la elevada incidencia que tiene este patrón de consumo en la adolescencia tardía y la juventud (EDADES, 2017; ESTUDES, 2014, 2016), ponen de manifiesto la necesidad de disponer de instrumentos que permitan identificar con precisión a aquellos jóvenes que se encuentran en mayor situación de riesgo, facilitando de este modo la intervención a realizar. Además, debido a la variedad de contextos en los que pueden ser evaluados los jóvenes (servicios de urgencias, Atención Primaria, gabinetes psicopedagógicos...), caracterizados muchos de ellos por la escasez de tiempo, un aspecto a valorar de las herramientas de screening será la brevedad y concisión.

Uno de los instrumentos más utilizados en la detección del consumo de riesgo ha sido el Alcohol Use Disorders Identification Test (AUDIT), sus tres primeros ítems -AUDIT-C- (Chung, Colby, Barnett, \& Monti, 2002; Thomas \& McCambridge, 2008; Seguel, Santander, \& Alexandre, 2013) e incluso tan solo el ítem número 3 AUDIT-3- (Bowring, Gouillou, Hellard, \& Dietze, 2013; Paiva et al., 2015). Al tratarse el BD de un patrón de consumo de riesgo, en un principio parecía lógico que se recurriese a estos mismos instrumentos para detectarlo, destacando el AUDIT-C como la mejor opción al ofrecer mayor sensibilidad y especificidad (Barry, Chaney, Stellefson \& Dodd, 2015; Cortés, Giménez, Motos \& Sancerni, 2016; Cortés, Giménez, Motos, Sancerni \&
Cadaveira, 2017; DeMartini \& Carey, 2012; Piano, Mazzuco, Kang, \& Phillips, 2017; Seth et al., 2015).

Sin embargo, la operacionalización cada vez más precisa de lo que se considera un BD/CIA ha evidenciado la necesidad de ajustar algunos de los ítems de este instrumento, buscando trasladar esta mejora a la identificación de estos jóvenes.

Entre las soluciones propuestas, por un lado, se ha desarrollado una línea de trabajo más conservadora en cuanto a la redacción del AUDIT, en la que se ha buscado aumentar la precisión del instrumento probando nuevas agrupaciones de sus ítems. McCambridge \& Thomas (2009), concluyeron que los ítems 3, 5 y 8 serían la mejor combinación. Posteriormente, Bowring et al. (2013), encontraron en población juvenil que la combinación de los ítems 3, 4, 8 y 9 por un lado; y los ítems 3, 4, 5 y 9 por otro, eran dos alternativas breves y válidas al AUDIT.

Por otra parte, la corriente más crítica con la forma de medir este patrón de consumo, ha optado por revisar las escalas de respuesta y/o la redacción de los ítems del AUDIT-C (García, Novalbos, Martínez, \& O'Ferrall, 2016; Higgins-Biddle \& Babor, 2018; Kokotailo et al., 2004). Las modificaciones más habituales en estos casos han sido la diferenciación de la cantidad consumida en función del sexo, la delimitación de la ocasión de consumo a unas dos horas e incluso la ampliación del periodo evaluado a los últimos seis meses para dar cuenta de la intermitencia de este patrón.

Teniendo en cuenta estos parámetros, Cortés et al. (2017) propusieron una nueva versión modificada de los ítems 2 y 3 del AUDIT-C denominada AR2I, adaptándola a una operacionalización del BD más precisa. Tras comparar la efectividad del AUDIT, AUDIT-C y AR2I, concluyeron que el mejor instrumento de screening para identificar a jóvenes BD era el AR2I, proponiéndose además un punto de corte 5 como el más indicado.

Pero no puede obviarse que este patrón de consumo está enmarcado dentro de un proceso adictivo (Kahler, Strong, \& Read, 2005; Pilatti, Caneto, Garimaldi, Vera, \& Pautassi, 2014) cuyo inicio y evolución es necesario atender. Para ello, una de las variables que cuenta con mayor apoyo experimental es la edad de inicio en el consumo de alcohol, pues evidencia la progresión de la ingesta de esta sustancia. Concretamente, se ha encontrado que a menor edad de 
inicio en el consumo mayor probabilidad de quedar inmerso en el proceso adictivo (Cortés \& Motos, 2015) y de experimentar mayor número de consecuencias derivadas del consumo (Pillai et al., 2014).

En suma, poder identificar a los jóvenes que presentan dentro de su consumo de alcohol un patrón de riesgo como lo es el BD, permitirá planificar medidas preventivas y de intervención ajustadas a sus necesidades, tanto desde el ámbito de Atención Primaria y Pediatría, como desde los Servicios de Urgencias y los especializados en prevención de conductas adictivas. Por este motivo, el objetivo de este trabajo es verificar qué propuesta basada en el AUDIT (versión completa, AUDITC, AR2I, agrupación de ítems 3-5-8, agrupación 3-4-8-9 y agrupación 3-4-5-9) constituye el mejor instrumento de screening en diferentes perfiles de jóvenes BD incluyendo una definición de consumidor de riesgo más amplia, que tiene en cuenta no sólo el consumo de 5 o más Unidades de Bebida Estándar -UBEs- en varones y 4 o más UBEs en mujeres durante un intervalo de dos horas, sino también los años que se lleva consumiendo alcohol.

\section{MÉTODO}

\section{Participantes}

El estudio ha contado con un total de 190 participantes, de los cuales 140 son mujeres (73.7\%) y 50 son hombres (26.3\%). Sus edades oscilan entre los $18 \mathrm{y}$ 22 años, con una media de edad de 18.79 años (DT=.98). La mayoría de los jóvenes reside en Valencia y zona metropolitana. Todos ellos son consumidores de alcohol, siendo la edad del primer consumo de esta sustancia los 14.56 años $(D T=1.39)$, sin existir diferencias estadísticamente significativas entre ambos sexos $\left(t_{(187)}=\right.$ $.538, p=.592)$.

\section{Instrumentos}

Se ha construido una batería con diferentes apartados:

Variables sociodemográficas. Incluye la edad y el sexo de los participantes.

Consumo de alcohol. Primeramente, se registra la edad del primer consumo de alcohol. Seguidamente, se rastrea tanto el consumo semanal como el realizado en un día especial mediante dos autoregistros. En el primero, los participantes anotan la cantidad y el tipo de alcohol ingerido, además de la hora aproximada en la que suelen realizar cada consumo. También especifican el número de meses que llevan consumiendo de esta manera. El autoregistro de ocasiones especiales incluye una tabla con las mismas variables, pero limitándolo a un día concreto, como un cumpleaños, fiestas locales, etc. También se recoge cuántas veces han realizado $\mathrm{CIA}$ durante los últimos 6 meses previos a la realización del pase. Para facilitarles la identificación del CIA se les muestra una tabla que les permite calcular las UBEs contenidas en las consumiciones que suelen ingerir.

A partir de la información ofrecida por los participantes, se han generado las siguientes variables:

Gramos máximos de alcohol consumidos. Teniendo en cuenta el autorregistro semanal y el día de consumo especial, se han traducido las consumiciones de los participantes a UBEs y posteriormente, a gramos de alcohol (1 UBE =10 gramos). Luego se han sumado los gramos totales de alcohol en cada episodio y se ha seleccionado de todos los episodios de consumo, aquel con mayor cantidad de gramos de alcohol ingeridos.

Realización o no de CIA. A partir del episodio de máximo consumo de alcohol, se ha clasificado a los participantes según si realizan una ingesta de alcohol en forma de CIA o no. Se ha utilizado una tabla de equivalencias para chicos y otra para chicas, donde se especifican las horas de consumo y los gramos necesarios que cada uno de ellos necesita para que se considere un episodio de $\mathrm{CIA}$.

Gramos máximos consumidos en un episodio de CIA. Se han sumado los gramos totales de alcohol consumidos durante un episodio de CIA. En los casos en los que el joven realiza más de un episodio de BD al día, se ha elegido aquel de mayor número de gramos de alcohol consumidos.

Horas de realización de CIA. Se han sumado las horas totales de duración del episodio de BD seleccionado.

Número de episodios de BD. Se han sumado los diferentes episodios de BD que han tenido lugar en un día de consumo de alcohol. 
Días de realización de $B D$ en los últimos 6 meses. Se han sumado los días totales de realización de BD en los últimos 6 meses.

Screening de consumo de riesgo. Se ha administrado el AUDIT, validado en población española por Guillamón, Gual \& Colom (1999). Este consta de 10 ítems, que aluden al patrón de consumo, la progresión hacia una dependencia y algunas consecuencias derivadas de la ingesta. La mayoría de ellos se responden con una escala Likert, con cinco opciones de respuesta: 0-Nunca, 1Menos de una vez al mes, 2- Mensualmente, 3Semanalmente y 4-A diario o casi a diario. Cuatro ítems presentan opciones de respuesta diferentes. Concretamente, el ítem 1 (0-Nunca, 1-Una vez al mes o menos, 2-De 2 a 4 veces al mes, 3-De 2 a 3 veces por semana y 4-Cuatro o más veces por semana), el ítem 2 (0Una o dos, 1- Tres o cuatro, 2- Cinco o seis, 3- De siete a nueve, 4- Diez o más) y los ítems 9 y 10, que tienen tres opciones de respuesta (0-No; 2-Sí, pero no durante el último año; 4-Sí, durante el último año). Esta prueba posee un buen nivel de consistencia interna $\alpha=.88$ (Guillamón et al., 1999).

AUDIT-C. A partir del AUDIT se ha calculado la puntuación para los tres primeros ítems, que dan lugar al AUDIT-C. Esta escala también presenta un coeficiente de fiabilidad bueno $\alpha=.84$ (Cortés et al., 2017).

AR2I. Se han introducido las mejoras de los ítems 2 y 3 propuestas por Cortés et al. (2017). Concretamente, el enunciado del ítem 2 se ha definido como: ¿Cuántas UBES sueles consumir en un día cualquiera en el que bebes alcohol?; respetándose la escala de respuesta original. El enunciado del ítem 3 quedó de la siguiente forma: Durante los últimos 6 meses ¿cuál es el número medio de días por mes en el que has consumido en dos horas 7 o más UBEs si eres varón o 6 o más UBEs si eres mujer? Para este ítem también se revisó su escala de respuesta: 0: Nunca; 1: Menos de una vez al mes; 2: Entre una y cuatro veces; 3: Entre cinco y ocho veces; 4: Entre nueve y doce veces; 5: Trece o más veces. La fiabilidad de esta nueva combinación de 2 ítems también es aceptable, $\alpha=.74$.

Esta nueva redacción del ítem 3 incluye las principales conclusiones derivadas de la investigación de los últimos años (Cortés \& Motos, 2015; Courtney \& Polish, 2009; Parada et al., 2011), presentando como la forma más precisa de medir el BD la inclusión de tres parámetros: la cantidad de gramos de alcohol ingeridos propuesta por el NIAAA en 2004 pero adaptando los gramos de alcohol a las UBEs de nuestro país, el intervalo temporal de ingesta fijado en una media de dos o tres horas y la duración mínima de realización de esta conducta fijada en los últimos 6 meses al tratarse de una conducta intermitente.

Por tanto, el AR2I contempla el CIA como la ingesta de 7 o más UBEs para los hombres (70 gramos o más) y 6 o más para las mujeres (60 gramos o más) durante un intervalo de 2-3 horas, al menos una vez en los últimos 6 meses.

En la muestra utilizada en este trabajo los coeficientes de fiabilidad, tanto en el AUDIT como del AUDIT-C y AR2I son similares, situándose en todos los casos entre .71 y .75. Las agrupaciones de ítems propuestas por otras investigaciones arrojan coeficientes de fiabilidad tan reducidos que nos han llevado a desestimar estas versiones en este trabajo (agrupación 35-8, $\alpha=.5$; agrupación 3-4-8-9, $\alpha=.45$; agrupación 3-4-5-9, $\alpha=.38)$.

\section{Procedimiento}

La muestra se recogió en las clases de primero y segundo curso de diferentes grados de la Universitat de València -Psicología, Logopedia, Criminología y Química-. En todos los casos, tras señalar el objetivo de la investigación y recalcar la voluntariedad y confidencialidad de los datos que se iban a facilitar, a los estudiantes que mostraron su disponibilidad se les indicó día y hora en la que debían asistir al laboratorio para la recogida de datos. Los pases tuvieron lugar durante los meses de diciembre de 2017 a marzo de 2018.

El día en el que se acudía al laboratorio los estudiantes rellenaban un consentimiento informado que incluía los objetivos de la investigación y la garantía de confidencialidad. Las entrevistas, supervisadas por los miembros del equipo de investigación, se realizaban en formato papel y de forma presencial, con la finalidad de resolver las dudas que pudieran surgir.

\section{Análisis de datos}

El procesamiento y análisis estadístico se ha llevado a cabo con el paquete SPSS 22 para Windows. 
Inicialmente, se ha realizado un análisis descriptivo de las distintas variables que forman parte de la investigación, con la finalidad de obtener las medias, las desviaciones típicas y los porcentajes de las mismas. A continuación, se ha realizado un ANOVA para observar si existen diferencias entre el grupo que consume en forma de BD y el que no, en cuanto a las puntuaciones obtenidas en las diferentes versiones del AUDIT.

Posteriormente, se ha llevado a cabo un análisis de conglomerados para determinar tipos de consumo de riesgo CIA de diferente intensidad. Para ello, se ha segmentado la muestra en función del sexo y se ha llevado a cabo un clúster bietápico, en donde las variables continuas han sido la edad de inicio en el consumo de alcohol y los gramos máximos de alcohol consumidos en un episodio CIA. Una vez hecho esto, se han realizado dos análisis de varianza (ANOVA) entre los grupos resultantes del conglomerado y las variables edad de inicio y gramos máximos de consumo de alcohol en un episodio CIA, con sus pruebas post hoc.

A partir de la clasificación obtenida, se ha realizado un análisis de varianza (ANOVA), con sus correspondientes pruebas a posteriori, considerando como variables independientes los grupos generados en el clúster, y las puntuaciones en las versiones del AUDIT como variables dependientes.

Además, se ha calculado el área bajo de la curva COR (Característica Operativa del Receptor) para observar cómo clasifican las distintas versiones del AUDIT a los consumidores CIA.

\section{RESULTADOS}

\section{Consumo de alcohol}

En general los jóvenes entrevistados consumen de media un máximo de 139.24 gramos de alcohol $(D T=79.54)$ en el día de mayor consumo, existiendo diferencias estadísticamente significativas en función del sexo $\left(t_{(65.84)}=3.86, p<.001\right)$, de modo que las mujeres consumen 124.11 gramos ( $D T=66.17)$ y los varones 181.58 gramos (DT=97.44).

Del total de los jóvenes, 107 realizan una ingesta de alcohol en forma de BD (56.3\%), consumiendo una media de 114.06 gramos de alcohol en cada episodio ( $D T=65.12)$.
En este caso, vuelven a ser los varones los que consumen en mayor medida $\left(t_{(33.35)}=2.99, p=.005\right)$ llegando a los 152.32 gramos ( $D T=86.98)$, frente a los 100.49 gramos de alcohol ingeridos por las jóvenes $(D T=49.31)$. En general, utilizan unas 2.93 horas en cada episodio de BD $(D T=1.44)$, aunque al comparar por sexos $\left(t_{(105)}=2.82, p=.006\right)$, las mujeres consumen en un intervalo de 2.71 horas (DT.= 1.23) mucho menor al utilizado por los varones que llega a alcanzar las 3.57 horas (DT=1.77). Por lo que respecta al número de episodios de BD a lo largo de un día, todos los entrevistados suelen realizarlo una vez $(M=1.15, D T=.41)$, no encontrándose diferencias significativas $\left(t_{(36.52)}=1.29\right.$, $p=.207$ ) en función del sexo. Finalmente, los participantes practican $\mathrm{BD}$ una media de 13.24 días $(D T=10.73)$ durante los últimos seis meses, sin encontrar diferencias en función del sexo $\left(t_{(188)}=1.19, p=.237\right)$.

\section{Versiones del AUDIT en función de realización o no de CIA}

El ANOVA realizado muestra que sí existen diferencias estadísticamente significativas entre los grupos BD y no BD en las puntuaciones de las tres versiones del AUDIT (Tabla 1).

Tabla 1. Versiones del AUDIT en función de realización de BD

\begin{tabular}{llccc}
\hline & & BD & No BD & F \\
\hline AUDIT & $M$ & 10.50 & 8.37 & $15.636^{* * *}$ \\
AUDIT-C & $D T$ & 3.26 & 4.14 & \\
& $M$ & 7.83 & 6.33 & $27.425^{* * *}$ \\
AR2I & $D T$ & 1.55 & 2.40 & \\
& $M$ & 5.76 & 4.42 & $41.242^{* * *}$ \\
& $D T$ & .85 & 1.93 & \\
\hline Nota: $B D=$ Binge Drinking; ${ }^{* * *} p<.001$ & & 83 &
\end{tabular}

\section{Grupos de consumidores CIA}

El análisis de conglomerados ha dado lugar a cinco grupos BD: tres de chicas y dos de chicos (Tabla 2). El primer grupo de chicas $\left(\mathrm{M}_{1}\right)$, formado por el $63.3 \%$ de las participantes, se ha iniciado casi a los 14 años en el consumo de alcohol y es el que ingiere menor número de gramos, aunque supera en casi 25 gramos el límite fijado en las mujeres para ser identificadas como BD. Las compañeras del segundo grupo $\left(\mathrm{M}_{2}\right)$, representan casi un tercio de las entrevistadas BD y consumen más de 40 gramos por encima del mínimo fijado. Finalmente, las chicas del tercer grupo $\left(\mathrm{M}_{3}\right)$, constituyen el porcentaje menor de consumidoras, pero con un consumo más 
excesivo. Por otro lado, el primer grupo de hombres $\left(\mathrm{H}_{1}\right)$ está formado por dos tercios de los BD y consumen un 50\% por encima de su límite. El segundo grupo de varones $\left(\mathrm{H}_{2}\right)$ lo forma el tercio restante de consumidores e ingieren tres veces más de este límite.

Tabla 2. Análisis de conglomerados de CIA en función del sexo

\begin{tabular}{lllllll}
\hline & $\mathbf{M}_{1}$ & $\mathbf{M}_{2}$ & $\mathbf{M}_{3}$ & $\mathbf{H}_{1}$ & $\mathbf{H}_{2}$ & $\mathbf{F}$ \\
\hline EIC & 13.68 & 15.96 & 13.0 & 15.41 & 13.40 & $37.95^{\text {** }}$ \\
& & & & & & \\
GCA & 84.10 & 102.83 & 228.17 & 109.41 & 226.50 & $32.54^{\text {** }}$ \\
& 50 & 23 & 6 & 17 & 10 & \\
$n$ & $(63.3 \%)$ & $(29.1 \%)$ & $(7.6 \%)$ & $(63 \%)$ & $(37 \%)$ & \\
\hline
\end{tabular}

Nota: $\mathrm{EIC}=$ Edad de inicio en el consumo de alcohol; GCA= Gramos máximos de consumo de alcohol en un episodio $\mathrm{CI} A ; \mathrm{M}_{1}=$ Agrupación de mujeres CIA con menor consumo de alcohol; $M_{2}=$ Agrupación de mujeres $\mathrm{CIA}$ con un consumo medio de alcohol; $\mathrm{M}_{3}=$ Agrupación de mujeres $\mathrm{CIA}$ con un consumo elevado de alcohol; $\mathrm{H}_{1}=$ Agrupación de varones $\mathrm{CIA}$ con un consumo medio de alcohol; $\mathrm{H}_{2}=$ Agrupación de varones $\mathrm{CIA}$ con un consumo de alcohol elevado; ${ }^{* * *} p<.001$

El ANOVA realizado para estudiar la edad de inicio en el consumo de alcohol según el tipo de ingesta realizada, indica que sí existen diferencias estadísticamente significativas entre los distintos grupos del análisis de conglomerados $\left(F_{(4,106)}=37.955, p<.001\right)$. Las pruebas post-hoc de Tukey ${ }^{1}$ señalan que los grupos medios tanto de varones como de mujeres $\left(\mathrm{M}_{2}\right.$ y $\left.\mathrm{H}_{1}\right)$ tienen una edad de inicio similar, diferenciándose ambos de manera significativa $(p<.001)$ del resto de grupos. Por otra parte, los dos grupos de consumidores BD elevados $\left(\mathrm{M}_{3}\right.$ y $\left.\mathrm{H}_{2}\right)$ también comparten la edad de inicio.

El ANOVA entre los gramos máximos de consumo de alcohol en un episodio BD y las tipologías de consumidores BD también indica que hay diferencias estadísticamente significativas $\left(\mathrm{F}_{(4,106)}=32.54, p<.001\right)$. En concreto, las pruebas post-hoc muestran que los dos grupos de mayor consumo (el de hombres $\mathrm{H}_{2}$ y el de mujeres $\mathrm{M}_{3}$ ) consumen significativamente más gramos que el resto de grupos $\mathrm{M}_{1}, \mathrm{M}_{2} \mathrm{y} \mathrm{H}_{1}(p<.001)$.

\section{Versiones del AUDIT según las tipologías de consumidores BD}

El ANOVA entre sujetos realizado para estudiar las puntuaciones de las versiones del AUDIT según el patrón de consumo BD (Tabla 3), indica que sí existen diferencias

\footnotetext{
1 Las pruebas post-hoc de Tukey que aparezcan en este trabajo se indicarán en una sola dirección, es decir, si las diferencias estadísticamente significativas están entre los grupos A y B, y entre los
}

entre los distintos grupos resultantes del análisis de conglomerados (AUDIT: $\mathrm{F}_{(4,106)}=3.725, p<.008$; AUDIT-C: $F_{(4,106)}=4.851, p<.002 ;$ AR21: $\left.F_{(4,106)}=4.05, p<.005\right)$.

Tabla 3. Puntuación en las diferentes versiones del AUDIT

\begin{tabular}{lcccccccc}
\hline & & $\mathrm{T}$ & $\mathrm{M}_{1}$ & $\mathrm{M}_{2}$ & $\mathrm{M}_{3}$ & $\mathrm{H}_{1}$ & $\mathrm{H}_{2}$ & $\mathrm{~F}$ \\
\hline AUDIT & $M$ & 10.50 & 10.92 & 8.65 & 13.33 & 10.24 & 11.40 & $3.725^{\text {** }}$ \\
& $D T$ & 3.27 & 2.81 & 3.27 & 2.25 & 3.61 & 3.72 & \\
AUDIT-C & $M$ & 7.83 & 7.94 & 6.83 & 9.33 & 8.00 & 8.40 & $4.851^{* * *}$ \\
& $D T$ & 1.56 & 1.43 & 1.37 & 1.51 & 1.22 & 2.01 & \\
AR2I & $M$ & 5.75 & 5.78 & 5.26 & 6.50 & 5.88 & 6.10 & $4.05^{* *}$ \\
& $D T$ & .85 & .86 & .69 & .84 & .70 & 0.88 & \\
\hline
\end{tabular}

Nota: $M=$ Media; $D T=$ Desviación típica; $T=T o t a l ; M_{1}=$ Agrupación de mujeres $\mathrm{CIA}$ con menor consumo de alcohol; $\mathrm{M}_{2}=$ Agrupación de mujeres $\mathrm{CIA}$ con un consumo medio de alcohol; $\mathrm{M}_{3}=$ Agrupación de mujeres $\mathrm{CI} A$ con un consumo elevado de alcohol; $\mathrm{H}_{1}=$ Agrupación de varones $\mathrm{CIA}$ con un consumo medio de alcohol; $\mathrm{H}_{2}=$ Agrupación de varones $\mathrm{CIA}$ con un consumo de alcohol elevado; ${ }^{* *} p<.01,{ }^{* * *} p \leq .001$

En cuanto a la versión original, las pruebas post-hoc de Tukey indican que el grupo $M_{2}$ puntúa significativamente por debajo de los grupos: $\mathrm{M}_{1}(p=.037)$ y $\mathrm{M}_{3}(p=.012)$.

Por lo que respecta al AUDIT-C, las pruebas post-hoc indican que las diferencias se encuentran entre los mismos grupos que la versión completa del instrumento, junto con el grupo $\mathrm{H}_{2}$. El grupo $\mathrm{M}_{2}$ tiene puntuaciones inferiores a las de los grupos $\mathrm{M}_{1}(p=.025), \mathrm{M}_{3}(p=.003)$ y $\mathrm{H}_{2}$ $(p=.041)$.

En la versión modificada (AR2I) se han encontrado diferencias entre los grupos $M_{2}$ y $M_{3}$. En este caso el grupo $M_{3}$ presenta una puntuación superior al $M_{2}$ $(p=.009)$.

\section{Curva COR}

Al diferenciar la muestra según realizan o no un consumo de alcohol en forma de BD, se encuentra que, tanto la versión original del AUDIT como la agrupación de los tres primeros ítems, muestran valores menores en el área bajo la curva COR en comparación con la versión modificada de este instrumento (Figura 1). En el caso del AUDIT, el área tiene un valor de .67; en el caso del AUDIT$C$ es de .69; y se incrementa hasta un valor de .72 para el AR2I.

grupos B y A, solo aparecerá indicada la primera de ellas, con la finalidad de no repetir la redacción de las mismas. 


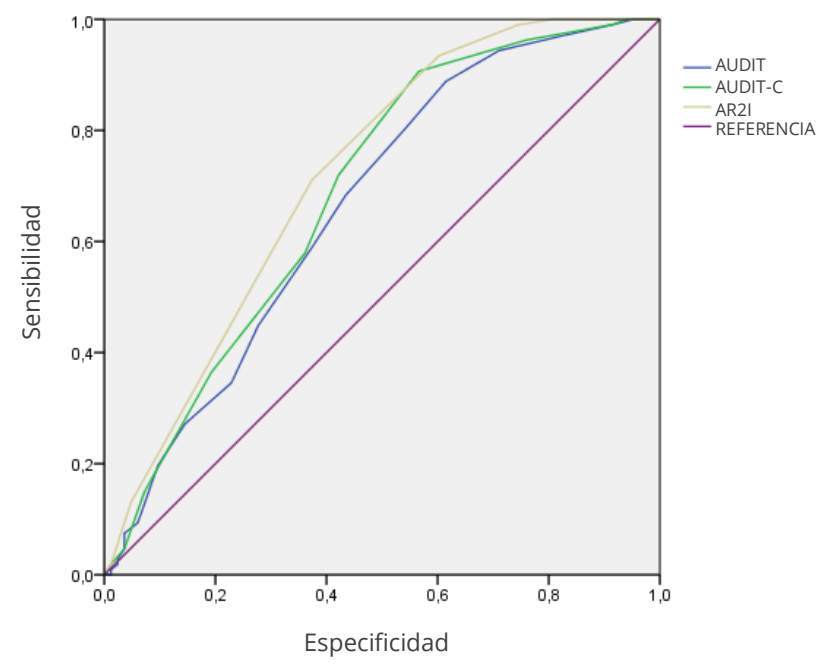

Figura 1. Curva Característica Operativa del Receptor (COR) para detectar el consumo en forma de BD en las distintas versiones del AUDIT

\section{DISCUSIÓN}

El objetivo principal de este trabajo es verificar qué versión del AUDIT se ajusta mejor como instrumento de screening del CIA/BD en jóvenes españoles, partiendo de una definición más precisa de este patrón de consumo, fruto de la investigación de los últimos años.

El BD es una práctica de riesgo que cada vez está más generalizada entre los jóvenes españoles. La epidemiología informa que aproximadamente 2 de cada 10 españoles realizan BD (EDADES, 2017), siendo en el tramo de edad entre los 20 y 24 años cuando este fenómeno alcanza una mayor representatividad (entre el $25 \%$ y $36 \%$, dependiendo del sexo). Si bien, los estudios epidemiológicos en población adolescente muestran una mayor prevalencia estando presente el BD en 6 de cada 10 adolescentes (ESTUDES, 2016), siendo a los 18 años cuando se constata una mayor prevalencia (entre el 54\% y 59\%, dependiendo del sexo). Con esto, los resultados de las encuestas epidemiológicas sitúan el BD como una práctica realizada entre la adolescencia tardía y el inicio de la juventud. Asimismo, también investigaciones nacionales e internacionales (Cortés et al., 2017; Pilatti, Read \& Caneto, 2015; Read, Haas, Radomski, Wickham, \& Borish, 2016), obtienen una incidencia muy elevada del $B D$ en jóvenes, por lo que puede afirmarse que el porcentaje de consumidores en forma de BD obtenido en este trabajo es similar al de estudios previos, siendo reflejo del patrón de consumo de alcohol habitual de los jóvenes de estas edades.

Es importante señalar que la ingesta de alcohol que realizan estos jóvenes está muy por encima del límite fijado en la operacionalización del BD. En general, tanto varones como mujeres llegan a doblar, e incluso triplicar, la cantidad de gramos de alcohol necesarios para ser catalogados como consumidores intensivos. Esta heterogeneidad de consumidores $\mathrm{BD}$ reafirma la necesidad de diferenciar grupos más homogéneos dentro de este colectivo que compartan un patrón de ingesta similar. Concretamente, en este estudio se identifican cinco grupos de consumidores BD, tres de mujeres y dos de hombres, en función de la edad en el inicio en el consumo de alcohol y los gramos ingeridos en cada episodio de BD.

Los dos grupos BD con un consumo de alcohol más elevado, uno de varones $\left(\mathrm{H}_{2}\right)$ y otro de mujeres $\left(\mathrm{M}_{3}\right)$, son los que agrupan menor número de jóvenes e iniciaron la ingesta de alcohol a edades más tempranas -alrededor de los 13 años-. En este caso, se observa una correspondencia entre la edad de inicio en el consumo de alcohol y los gramos de alcohol que se ingieren (Pillai et al., 2014), ya que este grupo que es el que presenta una menor edad de inicio es el que realiza un consumo mayor.

Además, al igual que en el estudio de Cortés et al. (2017), entre los grupos más intensos no se aprecian diferencias entre chicos y chicas en la intensidad de su consumo. Todos ellos llegan a triplicar la cantidad mínima de gramos de alcohol a partir de los cuales se identifica un BD. En este punto, no puede obviarse que realizar este mismo patrón de consumo supone un mayor riesgo para las mujeres, al ser más vulnerables a los efectos de esta sustancia psicoactiva (Arostegui \& Martínez, 2018).

También se encuentra equivalencia entre sexos entre los grupos de consumidores medios $\left(\mathrm{M}_{2}\right.$ y $\left.\mathrm{H}_{2}\right)$. En ellos el inicio en el consumo se produce entre los 15 y 16 años e ingieren la mitad de gramos de alcohol que sus compañeros más extremos, aunque también muy por encima de lo que se considera un episodio de BD para ambos.

Establecer grupos de BD permitirá perfilar con mayor precisión afirmaciones que se realizan para el colectivo genérico de jóvenes BD que redundarán en una 
atención profesional más precisa. Por ejemplo, cuando se habla a nivel general de la edad de inicio en el consumo de alcohol se concluye que entre varones y mujeres no se aprecian diferencias, pero tal como se observa en los resultados obtenidos estas son notorias cuando se establecen tipologías de consumo. En este caso, puede observarse que en los grupos medios y de mayor intensidad se mantiene una similitud en la edad de inicio entre varones y mujeres, aunque muy distante de lo que se propone en las encuestas epidemiológicas. No ocurre lo mismo en el caso de los colectivos más numerosos, donde las mujeres $\left(\mathrm{M}_{1}\right)$ muestran un inicio más temprano que los varones $\left(\mathrm{H}_{1}\right)$.

La misma reflexión puede hacerse en el caso de los gramos de consumo en un episodio BD. A nivel general, parece ser que los varones de las edades evaluadas consumen por encima de las mujeres (EDADES, 2017), pero no siempre es así tal como ha quedado patente en los grupos más intensos $\left(\mathrm{M}_{3}-\mathrm{H}_{2}\right)$ y de consumo medio $\left(\mathrm{M}_{2}-\right.$ $H_{1}$ ). Este resultado advierte de la homogeneidad en las cantidades consumidas entre los jóvenes, al tiempo que advierte de la importancia de incluir la perspectiva de género sin más demoras a la hora de abordar el BD. La evolución del patrón de consumo de alcohol entre las mujeres es preocupante, dado que éstas son más vulnerables a las consecuencias de la ingesta de esta sustancia (Arostegui \& Martínez, 2018; Wechsler \& Nelson, 2010). En este sentido, sería necesario analizar la influencia que ejercen las variables de género que intervienen en el consumo de alcohol entre chicos y chicas (roles culturales y sociales), ya que está demostrado que afectan en sus motivaciones hacia el consumo (Hussman, 2018).

Además, el consumo de riesgo en los jóvenes no puede quedar únicamente delimitado a los episodios de BD que llevan a cabo. Es cierto que a esta edad el BD constituye un patrón importante de consumo, pero no es el único. No contemplar la ingesta completa que realizan los jóvenes, tanto de manera intensiva como no, lleva a situaciones confusas como la de equiparar un consumidor de 90 gramos de alcohol con otro que hace este consumo y además a lo largo del día ingiere 90 gramos más, pero de manera no intensiva. De ahí la necesidad de tener en cuenta el consumo completo que realizan los $\mathrm{BD}$, contemplando además de los gramos de alcohol ingeridos de manera intensiva en el día de máximo consumo, alguna variable que refleje la globalidad de su consumo, como los gramos totales consumidos de forma no intensiva a lo largo de un día.

Esto permitiría explicar casos como el de la edad de inicio en el consumo de alcohol del grupo $\mathrm{M}_{1}$. Este colectivo se inicia de manera similar al de mujeres de máximo consumo, pero llama la atención que solo haga un consumo de 84 gramos de alcohol en cada episodio $\mathrm{CIA}$, mucho menor al de sus compañeras $\mathrm{M}_{3}$. Esto podría llevar a cuestionar la equiparación entre menor edad de inicio y mayor cantidad de consumo antes mencionada. Sin embargo, la situación cambia cuando se contemplan los gramos de alcohol consumidos independientemente de si se realizan dentro de un episodio de CIA o no. Este grupo (que es el mayoritario en mujeres) llega a consumir 150 gramos de manera no intensiva durante un día de consumo, cantidad muy superior a la ingesta durante un episodio de BD. No ocurre lo mismo en el grupo $\mathrm{M}_{2}$, en el que prácticamente coinciden los gramos de un episodio CIA (103 gramos) con los consumidos a lo largo de todo el día (128 gramos).

Llegados a este punto, cabe resaltar que la investigación no solo debe centrarse en la diferenciación de tipologías de consumidores BD, pues estaríamos dejando de atender a aquellos jóvenes cuyo patrón de consumo va mucho más allá de la mera ingesta puntual realizada en los episodios $\mathrm{BD}$. Se debería contemplar, además del BD, el consumo total de alcohol que realizan los jóvenes, lo que permitirá ubicarlos de manera precisa en la evolución de su propio proceso adictivo. De esta forma, se podrán llevar a cabo actuaciones de intervención adaptadas a las necesidades específicas de cada joven.

La elevada frecuencia del patrón de consumo BD entre los jóvenes y su demostrada heterogeneidad justifican la necesidad de disponer de instrumentos de screening que faciliten la identificación de este consumo de riesgo. Esto agilizaría cualquier intervención a realizar con ellos. En todas las versiones del AUDIT incluidas en este trabajo, los sujetos BD han puntuado por encima de los consumidores de alcohol que no realizan BD. Atendiendo a los puntos de corte propuestos por Cherpitel (1995) realizarían un consumo de riesgo tanto los BD como los noBD. Además, en este caso la desviación típica es tan grande que muchos de estos jóvenes podrían catalogarse erróneamente. El único de los tres instrumentos que identifica correctamente a la totalidad 
del colectivo BD sería el AR2I, mostrando un porcentaje muy reducido de noBD a los que se catalogaría erróneamente como posibles consumidores de riesgo. Este resultado es esperable, ya que la versión AR2I se realizó con la finalidad de adaptar sus ítems a una definición operacional de BD más precisa. Los resultados de esta investigación permiten sumar una evidencia más en favor de este instrumento al mostrarse más preciso en la identificación del BD en comparación con el AUDIT y AUDIT-C.

Al comparar la efectividad del AUDIT, AUDIT-C y AR2I para identificar posibles consumidores de riesgo en los diferentes grupos de BD se obtiene un resultado similar, tanto en los grupos de mujeres como de varones. Por ello, puede concluirse que el AR2I se define como la mejor opción para identificar consumidores de riesgo en colectivos BD. Con tan solo dos ítems, este instrumento permite detectar con mayor precisión al colectivo de consumidores intensivos. Además, su reducido tamaño facilita su inclusión en los recursos asistenciales de Atención Primaria, Pediatría, Servicios de Urgencias y recursos especializados en prevención de drogas, de manera que su aplicación en este tipo de servicios facilitará la detección temprana y la posterior derivación de los jóvenes BD a recursos asistenciales específicos, disminuyendo la probabilidad de que estos avancen en el proceso adictivo.

Por su parte, el que la consistencia interna de esta nueva combinación de ítems se califique como aceptable (.75) es un resultado esperable, ya que los ítems 2 y 3 han sido modificados para obtener dos aspectos diferentes del fenómeno BD. El ítem 2 toma en consideración la cantidad de alcohol ingerida durante un día de consumo, mientras que el ítem 3 mide la frecuencia de aparición del BD a través del paso del tiempo.

Finalmente, cabe destacar algunas limitaciones del presente estudio. El método de recogida de información sobre el patrón de consumo mediante autoinformes ha sido cuestionado en muestras adultas por presentar una infraestimación de esta conducta (Midanick, 1988; Smith, Remington, Williamson \& Anda, 1990). Sin embargo, también han demostrado ser herramientas fiables y válidas comparadas con otros protocolos (Babor, Kranzler \& Lauerman, 1989; Knight, Sherritt, Harris, Gates, \& Chang, 2003; Winters, Stinchfield, Henly, \& Schwartz, 1990) donde el sujeto percibe mayor riesgo de ser identificado (Darke, 1998; Degenhardt et al., 2013; Fowler \& Stringfellow, 2001; Williams, Toomey, McGovern, Wagenaar, \& Perry, 1995).

Una limitación adicional de este trabajo es la necesidad de evaluar un mayor número de varones de las diferentes intensidades de consumo BD. Esto permitiría establecer conclusiones más potentes al aumentar la representatividad de la muestra.

\section{REFERENCIAS}

Arostegui, E., \& Martínez, P. (2018). Mujeres y drogas. Manual para la prevención de recaídas con perspectiva de género. Bilbao: Instituto Deusto de Drogodependencias, Universidad de Deusto.

Babor, T. F., Kranzler, H. R., \& Lauerman, R. J. (1989). Early detection of harmful alcohol consumption: comparison of clinical, laboratory, and self-report screening procedures. Addictive Behaviors, 14(2), 139-157. doi:10.1016/0306-4603(89)90043-9

Barry, A. E., Chaney, B. H., Stellefson, M. L., \& Dodd, V. (2015). Evaluating the psychometric properties of the AUDIT-C among college students. Journal of Substance Use, 20(1), 1-5. doi:10.3109/14659891.2013.856479

Bowring, A. L., Gouillou, M., Hellard, M., \& Dietze, P. (2013). Comparing short versions of the AUDIT in a community-based survey of young people. BMC Public Health, 13(1), 301. doi:/10.1186/1471-2458-13$\underline{301}$

Cherpitel, C. J. (1995). Screening for alcohol problems in the emergency room: a rapid alcohol problems screen. Drug and alcohol dependence, 40(2), 133-137. doi:10.1016/0376-8716(95)01199-4

Chung, T., Colby, S. M., Barnett, N. P., \& Monti, P. M. (2002). Alcohol use disorders identification test: factor structure in an adolescent emergency department sample. Alcoholism: Clinical and Experimental Research, 26(2), 223-231. doi:10.1111/j.15300277.2002.tb02528.x

Cortés, M. T. (2010). Debates en drogodependencias: Aspectos sociales y de salud en el botellón: obligados a entenderse. Valencia: Plan Municipal de Drogodependencias. Ayuntamiento de Valencia.

Cortés, M. T., Giménez, J. A., Motos, P., \& Sancerni, M. D. (2016). Different versions of the Alcohol Use Disorders Identification Test (AUDIT) as screening instruments for underage binge drinking. Drug and Alcohol Dependence, 158(1), 52-59. doi:10.1016/j.drugalcdep.2015.10.033

Cortés, M. T., Giménez, J. A., Motos, P., Sancerni, M. D., \& Cadaveira, F. (2017). The utility of the Alcohol Use Disorders Identification Test (AUDIT) for the analysis of binge drinking in university students. Psicothema, 29(2), 229-235. doi:10.7334/psicothema2016.271

Cortés, M. T., \& Motos, P. (2015). Cómo definir y medir el Consumo Intensivo de Alcohol. En M. T. Cortés (Coord.), Consumo intensivo de 
alcohol en jóvenes (pp. 25-46). Barcelona, España: SOCIDROGALCOHOL.

Cortés, M. T., Motos, P., \& Giménez, J. A. (2015). Consecuencias bio-psicosociales derivadas del Consumo Intensivo de Alcohol: Aspectos psicosociales. En M. T. Cortés (Coord.), Consumo intensivo de alcohol en jóvenes (pp. 95-120). Barcelona, España: SOCIDROGALCOHOL.

Courtney, K. E., \& Polich, J. (2009). Binge drinking in young adults: Data, definitions, and determinants. Psychological bulletin, 135(1), 142156. doi:10.1037/ a0014414

Darke, S. (1998). Self-Report among injecting drug users: a review [see comments]. Drug and Alcohol Dependence, 51(3), 253-263. doi:10.1016/S0376-8716(98)00028-3

Degenhardt, L., O'Loughlin, C., Swift, W., Romaniuk, J. C., Coffey, C., Hall, W., \& Patton, G. (2013). The persistence of adolescent binge drinking into adulthood: findings from a 15-year prospective cohort study. BMJ Open, 3(8), 1-11. doi:10.1136/bmjopen-2013-003015

DeMartini, K. S., \& Carey, K. B. (2012). Optimizing the Use of the AUDIT for Alcohol Screening in College Students. Psychological Assessment, 24(4), 954-963. doi:10.1037/a0028519

ESPAD Group (2016). Results from the European School Survey Project on Alcohol and Other Drugs. Publications Office of the European Union, Luxembourg.

European Union (2010). Special Eurobarometer 331: EU citizens' attitudes towards alcohol. Brussels: EU.

Fowler, F. J., \& Stringfellow, V. L. (2001). Learning from experience: estimating teen use of alcohol, cigarettes, and marijuana from three survey protocols. Journal of Drug Issues. 31(3), 643-664.

García, M. A., Novalbos, J. P., Martínez, J. M.,...O'Ferrall, C. (2016). Validation of the Alcohol Use Disorders Identification Test in university students: AUDIT and AUDIT-C. Adicciones, 28(4), 194-204. doi:10.20882/adicciones.775

Guillamón, M., Gual, A., \& Colom, J. (1999). Test para la identificación de trastornos por uso de alcohol (AUDIT): Traducción y validación del AUDIT al catalán y castellano. Adicciones, 11(4), 337-347. doi:10.20882/adicciones.613

Higgins-Biddle, J. C., \& Babor, T. F. (2018). A review of the Alcohol Use Disorders Identification Test (AUDIT), AUDIT-C, and USAUDIT for screening in the United States: Past issues and future directions. The American journal of drug and alcohol abuse, 44(6), 578-586.

Hussman, J. B. (2018). The Pinking of Drinking: Understanding Women's Alcohol Use in Emerging Adulthood (tesis doctoral). Universidad de Toronto, Ontario, Canadá.

Kahler, C. W., Strong, D. R., \& Read, J. P. (2005). Toward efficient and comprehensive measurement of the alcohol problems continuum in college students: The brief young adult alcohol consequences questionnaire. Alcoholism: Clinical and Experimental Research, 29(7), 1180-1189. doi:10.1097/01.ALC.0000171940.95813.A5

Knight, J. R., Sherritt, L., Harris, S. K., Gates, E. C., \& Chang, G. (2003). Validity of brief alcohol screening tests among adolescents: a comparison of the AUDIT, POSIT, CAGE, and CRAFFT. Alcoholism Clinical \&
Experimental Research, 27(1), 67-73. doi:10.1111/j.15300277.2003.tb02723.x

Kokotailo, P. K., Egan, J., Gangnon, R., Brown, D., Mundt, M., \& Fleming, M. (2004). Validity of the Alcohol Use Disorders Identification Test in college students. Alcohol Clinical and Experimental Research, 28(6), 914-920. doi:10.1097/01.ALC.0000128239.87611.F5

López, E., Mota, N., Crego, A., Velásquez, T., Corral, M., Rodríguez, S., \& Cadaveira, F. (2014). Anomalías neurocognitivas asociadas al consumo intensivo de alcohol (binge drinking) en jóvenes y adolescentes: Una revisión. Adicciones, 26(4), 334-359. doi:10.20882/adicciones.39

Mallett, K. A., Marzell, M., Varvil-Weld, L., Turrisi, R., Guttman, K., \& Abar, C. (2011). One-time or repeat offenders? An examination of the patterns of alcohol-related consequences experienced by college students across the freshman year. Addictive Behaviors, 36(5), 508511. doi:10.1016/j.addbeh.2010.12.022

Martínez, J. A., Sher, K. J., \& Wood, P. K. (2014). Drinking consequences and subsequent drinking in college students over 4 years. Psychology of Addictive Behaviors, 28(4), 1240.

McCambridge, J., \& Thomas, B. A. (2009). Short forms of the AUDIT in a webbased study of young drinkers. Drug and Alcohol Review, 28(1), 18-24. doi: 10.1111/j.1465-3362.2008.00010.x

Midanick, L. (1988). The validity of self-report alcohol consumption and alcohol problems: a literature review. British Journal of Addiction banner, 77(4), 357-382. doi:10.1111/j.1360-0443.1982.tb02469.x

Motos, P. (2013). Determinantes del consumo intensivo de alcohol en jóvenes universitarios (tesis doctoral). Universidad de Valencia, Valencia, España.

Observatorio Español de la Droga y las Adicciones -OEDA- (2018). Encuesta sobre uso de drogas en Enseñanzas Secundarias en España (ESTUDES) 1994-2016. Madrid: Ministerio de Sanidad y Política Social (PNSD).

Observatorio Español de la Droga y las Adicciones-OEDA- (2019). Encuesta sobre Alcohol y drogas en España (EDADES) 1995-2017. Madrid: Ministerio de Sanidad y Política Social (PNSD).

Observatorio Español de la Droga y las Toxicomanías -OED- (2016). Encuesta sobre uso de drogas en Enseñanzas Secundarias en España (ESTUDES) 1994-2014. Madrid: Ministerio de Sanidad y Política Social (PNSD).

Parada, M., Corral, M., Caamaño-Isorna, F., Mota, N., Crego, A., Holguín, S. R., \& Cadaveira, F. (2011). Definición del concepto de consumo intensivo de alcohol adolescente (binge drinking). Adicciones, 23(1), 53-63. doi:10.20882/adicciones.167

Paiva, P. C. P., Paiva, H. N. D., Lamounier, J. A., Ferreira, E. F., César, C. A. S., \& Zarzar, P. M. (2015). Binge drinking among 12-year-old adolescent schoolchildren and its association with sex, socioeconomic factors and alcohol consumption by best friends and family members. Ciencia \& saude coletiva, 20(11), 3427-3435.

Piano, M. R., Mazzuco, A., Kang, M., \& Phillips, S. A. (2017). Binge Drinking Episodes in Young Adults: How Should We Measure Them in a 
Research Setting?. Journal of Studies on Alcohol and Drugs, 78(4), 502511. doi:10.15288/jsad.2017.78.502

Pilatti, A., Caneto, F., Garimaldi, J. A., Vera, B. V., \& Pautassi, R. M. (2014). Contribution of Time of Drinking Onset and Family History of Alcohol Problems in Alcohol and Drug Use Behaviors in Argentinean College Students. Alcohol and Alcoholism, 49(2), 128-137. doi:10.1093/alcalc/agt176

Pilatti, A., Read, J. P., \& Caneto, F. (2015). Validation of the Spanish Version of the Young Adult Alcohol Consequences Questionnaire (S-YAACQ). Psychological Assessment, 28(5), e49-e61. doi:10.1037/pas0000140

Pillai A., Nayak, M. B., Greenfield, T. K., Bond, J. C., Hasin, D. S., \& Patel, V. (2014). Adolescent drinking onset and its adult consequences among men: a population based study from India. Journal of Epidemiology and Community Health, 68(10), 922-927. doi:10.1136/jech-2014-204058

Read, J. P., Haas, A. L., Radomski, S., Wickham R. E., \& Borish, S. E. (2016). Identification of Hazardous Drinking With the Young Adult Alcohol Consequences Questionnaire: Relative Operating Characteristics as a Function of Gender. Psychological Assessment, 28(10), 1276-1289. doi:10.1037/pas0000251

Seguel-Palma, F., Santander-Manríquez, G., y Alexandre-Barriga, O. (2013). Validez y confiabilidad del test de identificación de los trastornos debidos al consumo de alcohol (AUDIT) en estudiantes de una universidad chilena. Ciencia y enfermería, 19(1), 23-35.

Seth, P., Glenshaw, M., Sabatier J. H. F., Adams, R., Du Preez, V., DeLuca, N., \& Bock, N. (2015). AUDIT, AUDIT-C, and AUDIT-3: Drinking Patterns and Screening for Harmful, Hazardous and Dependent Drinking in Katutura, Namibia. PLOS ONE, 10(3), 1-16. doi:10.1371/journal.pone.0120850

Shield, K. D., Gmel, G., Patra, J., \& Rehm, J. (2012). Global burden of injuries attributable to alcohol consumption in 2004: a novel way of calculating the burden of injuries attributable to alcohol consumption. Population Health Metrics, 10(1), 1-14. doi:10.1186/1478-7954-10-9

Smith, P. F., Remington, P. L., Williamson, D. F., \& Anda, R. F. (1990). A comparison of alcohol sales data with survey data on self-reported alcohol use in 21 states. American Journal of Public Health, 80(3), 309312. doi:10.2105/AJPH.80.3.309

Thomas, B. A., \& McCambridge, J. (2008). Comparative psychometric study of a range of hazardous drinking measures administered online in a youth population. Drug and Alcohol Dependence, 96(1-2), 121-127.

Wechsler, H., \& Nelson, T. F. (2001). Binge drinking and the American college students: What's five drinks? Psychology of Addictive Behaviors, 15(4), 287-291. doi:10.1037/0893-164X.15.4.287

Wechsler, H., \& Nelson, T. F. (2010). Will increasing alcohol availability by lowering the minimum legal drinking age decrease drinking and related consequences among youths? American journal of public health, 100(6), 986-992. doi:10.2105/AJPH.2009.178004

Williams, C. L., Toomey, T. L., McGovern, P. G., Wagenaar, A. C., \& Perry, C. L. (1995). Development, reliability, and validity of self-reported alcohol-use measures with young adolescents. Journal of Child \& Adolescent Substance Abuse, 4(3), 17-40. doi:10.1300/J029v04n03 02

Winters, K. C., Stinchfield, R. D., Henly, G. A., \& Schwartz, R. H.(1990). Validity of adolescent self-report of alcohol and other drug involvement. International Journal of the Addictions, 25(11), 13791395. doi:10.3109/10826089009068469 